\title{
Gravitational potential in Palatini formulation of modified gravity
}

\author{
Xin-He Meng ${ }^{1,2,3} * \quad$ Peng Wang ${ }^{1} \dagger$ \\ 1. Department of Physics, Nankai University, Tianjin 300071, P.R.China \\ 2. Institute of Theoretical Physics, CAS, Beijing 100080, P.R.China \\ 3. Department of Physics, University of Arizona, Tucson, AZ 85721
}

\begin{abstract}
General Relativity has so far passed almost all the ground-based and solar-system experiments. Any reasonable extended gravity models should consistently reduce to it at least in the weak field approximation. In this work we derive the gravitational potential for the Palatini formulation of the modified gravity of the $\mathrm{L}(\mathrm{R})$ type which admits a de Sitter vacuum solution. We conclude that the Newtonian limit is always obtained in those class of models and the deviations from General Relativity is very small for a slowly moving source.
\end{abstract}

Recently, some attempts have been made to explain the observed cosmic acceleration [1] by modifying the Einstein-Hilbert action [2-17]. Those include the $1 / R$ gravity [2] which may be predicted by String/M theory [3], the $1 / R+R^{2}$ [4] and $\ln R[5]$ gravity which intend to explain both the current acceleration and early time inflation. Generally, those models have two inequivalent formulations: the metric formulation (second order formulation) and the Palatini formulation (first order formulation) 6, 7, 8, 9, 10. There are many interesting features in the Palatini formulation of those models, e.g. the absence of the instability [8] appeared in the metric formulation [16] (However, this instability in metric formulation may be resolved by a $R^{2}$ term [4] or conformal anomaly induced terms [17]), the universality of the field equations for vacuum [18] or source with constant trace of the energymomentum tensor [19]. There is also a third alternative to the above two formulations [11, 12]. In those works, it has been shown that the simplest proposal of $1 / R$ gravity in metric formulation is in conflict with solar system gravitational experiments [13] and the Palatini formulation is in conflict with electron-electron scattering experiments 7]. But it is still worth continuing to explore whether some phenomenological model of this type or its variants can easily accommodate the data. Thus it is suitable to explore some basic features of this type of models at this time. Specifically, any reasonable gravity theory should reduce to Newton gravity for slowly moving weak source. The condition of having a Newtonian limit for the metric formulation is explored in Ref.[14]. In this paper, we will discuss the weak field expansion of Palatini formulation. We will follow the sign conventions of Ref. 7].

In general, when handled in Palatini formulation, one considers the action to be a functional of the metric $\bar{g}_{\mu \nu}$ and a connection $\hat{\nabla}_{\mu}$ which is independent of the metric. The resulting modified gravity action can be written as

$$
S\left[\bar{g}_{\mu \nu}, \hat{\nabla}_{\mu}\right]=\frac{1}{2 \bar{\kappa}^{2}} \int d^{4} x \sqrt{-\bar{g}} L(\hat{R})+S_{M},
$$

where $\bar{\kappa}$ is a constant with dimension (mass) ${ }^{-1}$ that will be specified below, $\hat{R}_{\mu \nu}$ is the Ricci tensor of the connection $\hat{\nabla}_{\mu}, \hat{R}=\bar{g}^{\mu \nu} \hat{R}_{\mu \nu}$ and $S_{M}$ is the matter action. For the theory (1) to explain the current cosmic acceleration, the vacuum solution is necessarily de Sitter. Thus, when studying its Newtonian limit, we must expand around de Sitter background. The Newtonian limit of the metric formulation of this sort of modified gravity was studied in Ref. 14] and it concluded that the existence of a weak field approximation around the de Sitter background can be achieved by imposing the condition $L^{\prime \prime}\left(R_{0}\right)=0$ where $R_{0}$ is the vacuum solution in metric formulation. All the current existed model that may explain the current cosmic acceleration, namely, the $1 / R$ and $\ln R$ model, do not satisfy this condition. And it is shown in Ref. [7] that the condition for the existence of an equivalent scalar-tensor description of the theory (1) is just $L^{\prime \prime} \neq 0$. The absence of such an equivalent description is a strong indication that the original theory is unphysical [20]. Thus if the condition for the existence of Newtonian limit in Palatini formulation is the same as the metric formulation, the whole framework of explaining cosmic acceleration in Palatini formulation is doubtable. Fortunately, as we will show in

\footnotetext{
*xhmeng@phys.nankai.edu.cn

† pewang@eyou.com
} 
this work. This is not the case. The theory (1) always has Newtonian limit and the deviations from the Newtonian potential with a standard Yukawa term (since we are working in de Sitter background) are very small for a slowly moving source.

First, let us give the field equations of (1). See Ref. [6] for details.

Varying the action with respect to $\bar{g}_{\mu \nu}$ gives

$$
L^{\prime}(\hat{R}) \hat{R}_{\mu \nu}-\frac{1}{2} L(\hat{R}) \bar{g}_{\mu \nu}=\bar{\kappa}^{2} T_{\mu \nu}
$$

where $T_{\mu \nu}$ is the energy momentum tensor and is given by

$$
T_{\mu \nu}=-\frac{2}{\sqrt{-\bar{g}}} \frac{\delta S_{M}}{\delta \bar{g}^{\mu \nu}} .
$$

Varying with respect to the connection, one can find that $\hat{R}_{\mu \nu}$ is related to $\bar{R}_{\mu \nu}$ by

$$
\hat{R}_{\mu \nu}=\bar{R}_{\mu \nu}+\frac{3}{2}\left(L^{\prime}\right)^{-2} \bar{\nabla}_{\mu} L^{\prime} \bar{\nabla}_{\nu} L^{\prime}-\left(L^{\prime}\right)^{-1} \bar{\nabla}_{\mu} \bar{\nabla}_{\nu} L^{\prime}-\frac{1}{2}\left(L^{\prime}\right)^{-1} g_{\mu \nu} \bar{\square} L^{\prime}
$$

and contracting with $\bar{g}_{\mu \nu}$ gives

$$
\hat{R}=\bar{R}+\frac{3}{2}\left(L^{\prime}\right)^{-2}\left(\bar{\nabla}_{\mu} L\right)^{2}-3\left(L^{\prime}\right)^{-1} \bar{\square} L^{\prime}
$$

where $\bar{R}_{\mu \nu}$ is the Ricci tensor with respect to $\bar{g}_{\mu \nu}$ and $\bar{R}=\bar{g}^{\mu \nu} \bar{R}_{\mu \nu}$.

By contracting Eq.(2) with $\bar{g}_{\mu \nu}$, we can solve $\hat{R}$ as a function of $T$ :

$$
L^{\prime}(\hat{R}) \hat{R}-2 L(\hat{R})=\bar{\kappa}^{2} T
$$

where a prime denotes differentiation with respect to $\hat{R}$. Thus (4), (5) do define the Ricci tensor with respect to $\hat{\nabla}_{\mu}$ and $L(\hat{R})$ in Eq.(2) is actually a function of $T$. Specifically, we denote the vacuum solution by $\hat{R}_{0}=R_{0}$ and thus $\hat{R}_{\mu \nu}^{(0)}=\bar{g}_{\mu \nu} R_{0} / 4$. By Eq.(4), $\bar{R}_{\mu \nu}^{(0)}=\hat{R}_{\mu \nu}^{(0)}$ and thus $\bar{R}_{0}=\hat{R}_{0}=R_{0}$.

The aim of this paper is to treat the approximation in which gravity is "weak". In the context of modified gravity, this means that the spacetime metric is nearly de Sitter. In application of gravity theory, this is an excellent approximation except for phenomena dealing with strong gravitational fields such as black holes and large scale structure of the universe. Thus in presence of some source, we divide the metric into two parts

$$
\bar{g}_{\mu \nu}=\bar{g}_{\mu \nu}^{(0)}+h_{\mu \nu}
$$

where $\bar{g}_{\mu \nu}^{(0)}$ is the de Sitter vacuum solution of the field equations and $h_{\mu \nu}$ represents deviations which vanish at infinity. The first order variation of $\bar{R}_{\mu \nu}$ is given by the Palatini identity

$$
\delta \bar{R}_{\mu \nu}=\frac{1}{2}\left(\bar{\nabla}_{\mu} \bar{\nabla}^{\rho} h_{\rho \nu}+\bar{\nabla}_{\nu} \bar{\nabla}^{\rho} h_{\rho \mu}\right)+\frac{1}{3} R_{0} h_{\mu \nu}-\frac{1}{12} R_{0} \bar{g}_{\mu \nu}^{(0)} h-\frac{1}{2} \bar{\square}_{\mu \nu}-\frac{1}{2} \bar{\nabla}_{\mu} \bar{\nabla}_{\nu} h
$$

where $h=h_{\mu \nu} \bar{g}^{(0) \mu \nu}$.

Since in the vacuum, we have $\hat{R}_{\mu \nu}^{(0)}=\bar{R}_{\mu \nu}^{(0)}$, the relation between perturbations of those two quantities can be read off from Eq.(4):

$$
\delta \hat{R}_{\mu \nu}=\delta \bar{R}_{\mu \nu}+\frac{3}{2}\left(L^{\prime}\right)^{-2} \bar{\nabla}_{\mu} L^{\prime} \bar{\nabla}_{\nu} L^{\prime}-\left(L^{\prime}\right)^{-1} \bar{\nabla}_{\mu} \bar{\nabla}_{\nu} L^{\prime}-\frac{1}{2}\left(L^{\prime}\right)^{-1} \bar{g}_{\mu \nu}^{(0)} \bar{\square} L^{\prime}
$$

and contracting with $\bar{g}^{(0) \mu \nu}$ gives

$$
\delta \hat{R}=\delta \bar{R}+\frac{3}{2}\left(L^{\prime}\right)^{-2}\left(\bar{\nabla}_{\mu} L\right)^{2}-3\left(L^{\prime}\right)^{-1} \bar{\square} L^{\prime}
$$

A subtlety in expansion with respect to de Sitter background is that $\hat{R} \neq R_{0}+\delta \hat{R}$, instead, by writing $\hat{R}=\left(\bar{g}^{(0) \mu \nu}+h^{\mu \nu}\right)\left(\hat{R}_{\mu \nu}^{(0)}+\delta \hat{R}_{\mu \nu}\right)$ explicitly, we can find that $\hat{R}=R_{0}-\frac{R_{0}}{4} h+\delta \hat{R}_{\mu \nu}$, where 
$h=h_{\mu \nu} \bar{g}^{(0) \mu \nu}$. Without the $h$ term, the resulting equation will not be gauge-invariant. Specifically, this term should be added to the expansion equation in Ref. [14], but its conclusion is unchanged.

The first order expansion of Eq.(2) with respect to the de Sitter vacuum solution $\bar{R}^{(0)}$ gives

$$
\begin{aligned}
L^{\prime}\left(R_{0}\right) \delta \hat{R}_{\mu \nu}+ & \frac{1}{4}\left[L^{\prime \prime}\left(R_{0}\right) R_{0}-2 L^{\prime}\left(R_{0}\right)\right] \bar{g}_{\mu \nu}^{(0)} \delta \hat{R}-\frac{1}{2} L\left(R_{0}\right) h_{\mu \nu} \\
& +\left[\frac{R_{0} L^{\prime}\left(R_{0}\right)}{8}-\left(\frac{R_{0}}{4}\right)^{2} L^{\prime \prime}\left(R_{0}\right)\right] \bar{g}_{\mu \nu}^{(0)} h=\bar{\kappa}^{2} T_{\mu \nu}
\end{aligned}
$$

By Eq.(9) and the fact that $L^{\prime}(\hat{R})$ is actually a function of $T$ we can move all the "source" term in the above equation to the right-hand side

$$
\begin{aligned}
& L^{\prime}\left(R_{0}\right) \delta \bar{R}_{\mu \nu}+\frac{1}{4}\left[L^{\prime \prime}\left(R_{0}\right) R_{0}-2 L^{\prime}\left(R_{0}\right)\right] \bar{g}_{\mu \nu}^{(0)} \delta \bar{R}-\frac{1}{2} L\left(R_{0}\right) h_{\mu \nu} \\
& \quad+\left[\frac{R_{0} L^{\prime}\left(R_{0}\right)}{8}-\left(\frac{R_{0}}{4}\right)^{2} L^{\prime \prime}\left(R_{0}\right)\right] \bar{g}_{\mu \nu}^{(0)} h=\bar{\kappa}^{2} T_{\mu \nu}+L^{\prime}\left(R_{0}\right) S_{\mu \nu}
\end{aligned}
$$

where $S_{\mu \nu}$ is a function of $\bar{\nabla}_{\mu} T$ and $\bar{\nabla}_{\mu} \bar{\nabla}_{\nu} T$ given by

$$
\begin{aligned}
S_{\mu \nu}= & \left(L^{\prime}\right)^{-1} \bar{\nabla}_{\mu} \bar{\nabla}_{\nu} L^{\prime}+\frac{1}{2}\left(L^{\prime}\right)^{-1} \bar{g}_{\mu \nu}^{(0)} \bar{\square} L^{\prime}-\frac{3}{2} \bar{\nabla}_{\mu} L^{\prime} \bar{\nabla}_{\nu} L^{\prime} \\
& -\frac{3}{4}\left[\frac{L^{\prime \prime}\left(R_{0}\right) R_{0}}{L^{\prime}\left(R_{0}\right)}-2\right] \bar{g}_{\mu \nu}^{(0)}\left[\frac{1}{2}\left(L^{\prime}\right)^{-2}\left(\bar{\nabla}_{\mu} L\right)^{2}-L^{\prime} \bar{\square} L^{\prime}\right]
\end{aligned}
$$

It is easy to see that the choice of $L(R)=R-2 \Lambda$ reproduces the expansion equation of General Relativity around de Sitter background [22], which is natural since now the Palatini formulation is equivalent to the metric formulation. But note that this is the most general Lagrangian that those two formulations are equivalent.

Let us consider the gauge transformation

$$
h_{\mu \nu} \longrightarrow h_{\mu \nu}+\bar{\nabla}_{\mu} \xi_{\nu}+\bar{\nabla}_{\nu} \xi_{\mu}
$$

where $\xi_{\mu}$ is an arbitrary vector field. By equation $\triangle_{\xi} \delta \bar{R}_{\mu \nu}=\frac{R_{0}}{4}\left(\bar{\nabla}_{\mu} \xi_{\nu}+\bar{\nabla}_{\nu} \xi_{\mu}\right)$, it is not hard to check that Eq.(12) is invariant under this gauge tansformation. Thus we can choose a suitable $\xi_{\mu}$ to impose the transverse-traceless (TT) gauge on $h_{\mu \nu}$

$$
\bar{\nabla}^{\mu} h_{\mu \nu}=0, h=0 .
$$

This gauge-invariance also implies that the graviton described by theory (1) is still massless.

After gauge fixing, from Eq. (8), the expansion equation (14) can be written in a much more simplified and illuminating form

$$
\bar{\square} h_{\mu \nu}-\frac{R_{0}}{6} h_{\mu \nu}=\frac{-2 \bar{\kappa}^{2}}{L^{\prime}\left(R_{0}\right)} T_{\mu \nu}-2 S_{\mu \nu}
$$

If we identify the $\bar{\kappa}^{2}$ appearing in action (1) as $\kappa^{2} / L^{\prime}\left(R_{0}\right)$, where $\kappa^{2}=8 \pi G$ and $G$ is the Newton constant, this equation is identical to the expansion equation of General Relativity around a de Sitter background except the appearance of the $S_{\mu \nu}$ term. Note that for any reasonable model, $L^{\prime}\left(R_{0}\right) \sim 1$, thus $\bar{\kappa}$ is actually the same order of magnitude as $\kappa$. Since $S_{\mu \nu}$ depends only on the derivatives of $T$, for the vacuum or a constant $T$, Eq.(16) reduces exactly to the expansion equation of General Relativity. This confirms in another way the conclusion that the theory (1) will reduce to General Relativity in the case of vacuum [18] and constant $T$ [19] in an unified and illuminating way.

Thus for a slowly varying source, denoting its density by $\rho=-T$, the Newtonian potential it generates will be

$$
\Phi(r)=\frac{G \rho-S_{00} / 16 \pi}{r} \exp \left(-\sqrt{\frac{R_{0}}{6}} r\right)
$$

The exponential is just the standard Yukawa term in the gravitational potential in de Sitter background in General Relativity. With $R_{0} \sim H_{0}^{2}$, where $H_{0}$ is the current Hubble parameter and is given by 
$H_{0} \simeq 100 \mathrm{~km} / \mathrm{s} . M p c$, the effects of the Yukawa term can be neglected in solar system or ground-based gravitational experiments. All the effects of the modified action in Palatini formulation are enclosed in the $S_{00}$ term. This is the secret of the modified gravity models in Palatini formulation.

Since now the form of Newtonian potential has been severely constrained by experiments (see Ref. 21] for a review), the effects of $S_{00}$ should be extremely small to evade the current constraints. Let us now estimate its magnitude for the class of models that intend to explain the current cosmic acceleration. For those models, we generally have $L(\hat{R})=\hat{R}+f(\hat{R})$. In order to explain current acceleration and reduce to General Relativity at early times, it requires that when $\hat{R} \sim H_{0}^{2}$, the $f(\hat{R})$ term dominates, when $\hat{R} \gg H_{0}^{2}$, the $\hat{R}$ term dominates. Any test objects in current gravitational experiments satisfy $\kappa^{2} \rho \gg H_{0}^{2}$, e.g. for an object with density of the order $10^{3} \mathrm{~kg} / \mathrm{m}^{3}, \kappa^{2} \rho / H_{0}^{2} \sim 10^{29}$. Thus in those cases, $L(\hat{R}) \sim \hat{R} \sim \kappa^{2} \rho$ and $f(\hat{R}) \ll \hat{R}$. Then

$$
\begin{array}{r}
L^{\prime} \sim 1+f(\hat{R}) / \hat{R} \sim 1, \quad \bar{\nabla}_{0} L^{\prime}=f^{\prime \prime}(\hat{R}) \kappa^{2} \dot{\rho} \sim \frac{f(\hat{R})}{\hat{R}} \frac{\dot{\rho}}{\rho}, \\
\bar{\nabla}_{0} \bar{\nabla}_{0} L^{\prime}=f^{\prime \prime}(\hat{R}) \kappa^{2} \ddot{\rho}+f^{\prime \prime \prime}(\hat{R})\left(\kappa^{2} \dot{\rho}\right)^{2} \sim \frac{f(\hat{R})}{\hat{R}} \frac{\ddot{\rho}}{\rho}+\frac{f(\hat{R})}{\hat{R}}\left(\frac{\dot{\rho}}{\rho}\right)^{2}
\end{array}
$$

Thus we can see from Eq.(13) that all the terms in $S_{00}$ are suppressed by a $f(\hat{R}) / \hat{R}$ factor, which is in practice the order $H_{0}^{4} /\left(\kappa^{2} \rho\right)^{2} \sim 10^{-58}$ that is extremely small for any test objects in current gravitational experiments. Thus the Newtonian limit is always obtained. The above estimate is quite obvious in a specific example: the $1 / R$ gravity [2]. There, $L(\hat{R})=\hat{R}+\alpha^{2} / \hat{R}$, where $\alpha \sim H_{0}^{2}$. $\hat{R}$ is related to $\rho$ trough Eq.(5) and is given by

$$
\hat{R}=\frac{1}{2}\left[\bar{\kappa}^{2} \rho+\sqrt{4 \alpha^{2}+\left(\bar{\kappa}^{2} \rho\right)^{2}}\right]
$$

The above computations and conclusions are also easily extended to the matter loops corrected modified gravity in Palatini formulation [12]. This type of models can be written as

$$
S_{\text {loop }}\left[\bar{g}_{\mu \nu}, \hat{\nabla}_{\mu}\right]=\frac{1}{2 \bar{\kappa}^{2}} \int d^{4} x \sqrt{-\bar{g}}[\bar{R}+f(\hat{R})] \equiv S_{E H}+S_{\text {Palatini }},
$$

where $S_{E H}$ is the familiar Einstein-Hilbert action. When written in the Einstein frame, the presence of a $\bar{R}$ term will induce a kinetic term for the scalar field.

The counterpart of Eq.(11) is

$$
\begin{array}{r}
\delta \bar{R}_{\mu \nu}+f^{\prime}\left(R_{0}\right) \delta \hat{R}_{\mu \nu}+\frac{1}{4}\left[f^{\prime \prime}\left(R_{0}\right) R_{0}-2 f^{\prime}\left(R_{0}\right)\right] \bar{g}_{\mu \nu}^{(0)} \delta \hat{R}-\frac{1}{2}\left[R_{0}+f\left(R_{0}\right)\right] h_{\mu \nu} \\
+\left[\frac{R_{0} f^{\prime}\left(R_{0}\right)}{8}-\left(\frac{R_{0}}{4}\right)^{2} f^{\prime \prime}\left(R_{0}\right)\right] \bar{g}_{\mu \nu}^{(0)} h=\bar{\kappa}^{2} T_{\mu \nu}
\end{array}
$$

Then following the same line of analysis above, we can see that $\bar{\kappa}^{2}=\kappa^{2} /\left(1+f^{\prime}\left(R_{0}\right)\right)$ and the gravitational potential is also given by Eq.(17) with the $L^{\prime}$ in the expression for $S_{\mu \nu}$ replaced by $f^{\prime}$. Thus in particular, the model (20) will reduce to General Relativity in the case of vacuum or source with constant trace of energy-momentum tensor.

In conclusion, we computed the gravitational potential by weak field expansion of Palatini formulation of modified gravity which admits a de Sitter vacuum solution. We conclude that the Newtonian limit is always obtained in those class of models and the deviations from General Relativity is very small for slowly moving source. It is well-known that there are some motivations to extend General Relativity, such as its incompatibility with quantum mechanics, non-localities and unavoidable singularities in its solutions. In the recent works the main motivation is to explain the recently observed cosmic acceleration without dark energy. Although the proposal of a $1 / R$ term is doomed now. It is still worth continuing to explore whether some phenomenological model of this type or its variants can easily accommodate the data. There is an observation in this direction of model-building: To drive an current cosmic acceleration, one only need the a de Sitter vacuum solution and in order to reduce to General Relativity, $L(R) \rightarrow R$ when $R / H_{0}^{2} \gg 1$. Specifically, it is not necessary to have $L(R) \rightarrow \infty$ as $R \rightarrow 0$ which may introduce instabilities. 


\section{Acknowledgements}

We would especially like to thank Sergei Odintsov for a careful reading of the manuscript and helpful comments. We also wish to thank É.É.Flanagan and S.Nojiri for helpful discussions. This work is partly supported by China NSF, Doctoral Foundation of National Education Ministry and ICSC-World lab. scholarship.

[1] S. Perlmutter el al. Nature 404 (2000) 955; Astroph. J. 517 (1999) 565; A. Riess et al. Astroph. J. 116 (1998) 1009; ibid. 560 (2001) 49; Y. Wang, Astroph. J. 536 (2000) 531; D.N.Spergel, et al., astro-ph/0302207 L.Page et al. astro-ph/0302220 M.Nolta, et al, astro-ph/0305097. C.Bennett, et al, astro-ph/0302209.

[2] S.M.Carroll, V.Duvvuri, M.Trodden and M. Turner, astro-ph/0306438 S. Capozziello, S. Carloni and A. Troisi, "Recent Research Developments in Astronomy \& Astrophysics" -RSP/AA/21-2003 astro-ph/0303041; S.Capozziello, Int.J.Mod.Phys.D 11 (2002) 483;

[3] S.Nojiri and S.D.Odintsov, hep-th/0307071

[4] S.Nojiri and S.D.Odintsov, hep-th/0307288

[5] S.Nojiri and S.D.Odintsov, hep-th/0308176

[6] D. N. Vollick, astro-ph/0306630

[7] É.É.Flanagan, astro-ph/0308111

[8] X.H.Meng and P.Wang, to appear in Class. and Quant. Grav. astro-ph/0307354; ibid, astro-ph/0308031

[9] X.H.Meng and P.Wang, astro-ph/0308284

[10] X.H.Meng and P.Wang, hep-th/0309062

[11] É.É.Flanagan, gr-qc/0309015

[12] X.H.Meng and P.Wang, hep-th/0310038

[13] T.Chiba, astro-ph/0307338

[14] R.Dick, gr-qc/0307052

[15] M. E. Soussa and R. P. Woodard, astro-ph/0308114

[16] A. D. Dolgov and M. Kawasaki, astro-ph/0307285

[17] S.Nojiri and S.D.Odintsov, hep-th/0310045

[18] M.Ferraris, M.Francaviglia and I.Volovich, Nouvo Cim. B108 (1993) 1313 gr-qc/9303007; ibid, Class. Quant.Grav. 11 (1994) 1505;

[19] G.F.Rubilar, Class. Quant. Grav. 15 (1998) 239;

[20] G.Magnano and L.M.Sokolowski, Phys.Rev.D 50 (1994) 5039 gr-qc/9312008;

[21] C. M. Will, Theory and Experiment in Gravitational Physics, Cambridge Universtiy Press, Cambridge, 1993; Living Rev. Rel. 4 (2001) 4;

[22] A. Higuchi, Nucl. Phys. B 325 (1989) 745; 